beginning the pharmaceuticals industry was run by chemists. This was not so bad . . Now most of them are run by people with MBAs ... people who could be the chief executive of Renault, Volvo or anything. They don't know anything about drugs". The problem comes when a chemist presents an interesting drug to the financial analyst, who asks: "What is the market?" The chemist has to decide for what indication the drug will be developed. If the indication is not there, it must be created.

One of many examples of this process was the development in the 1970s of alprazolam (Xanax) for panic disorder. According to David Sheehan (Institute for Research and Psychiatry, Tampa, Florida), the marketing of this drug involved a "clear strategy" to take advantage of the medical profession's confusion in the classification of anxiety disorders; "to create a perception that the drug had special and unique properties that would help it capture market share and displace diazepam from the top position ... There was in fact nothing unique in this regard about Xanax ... benzodiazepines were all good for panic disorder". Xanax was marketed by Upjohn with Food and Drug Administration approval of doses up to $6 \mathrm{mg}$ daily (equivalent to $60-120 \mathrm{mg}$ diazepam). It is perhaps no coincidence, as Healy observes, that the effective incidence of panic disorders has grown a thousand-fold since 1980 .

Sheehan relates a similar 'deliberate tactic' used to market $5-\mathrm{HT}_{1 \mathrm{~A}}$ agonists by generating hysteria in the medical profession about the dangers of benzodiazepines. The $5-\mathrm{HT}_{1 \mathrm{~A}}$ agonists were not a great success, but were succeeded by the selective serotonin reuptake inhibitors (SSRIs), which seemed to show activity in a range of anxiety disorders. Interestingly, according to Healy, the effective incidence of depression, obsessive-compulsive disorder, social phobia, and post-traumatic stress disorder has also increased a thousand-fold since 1980. There are now a number of 'me too' SSRIs because they are cheaper to make than new drugs. Fridolin Sulser of Vanderbilt University notes: "If you can get $20 \%$ of fluoxetine's market, you can make 400-500 million dollars a year with very little investment in research and development". He adds, sadly: "I don't know how to solve this dilemma in an industrial society that is so heavily driven by profits."

Other sinister tales about the machinations of drug companies abound. Ian Oswald describes his well-known battle to get his paper about Upjohn's drug triazolam (Halcion) published, only to find that the then editor of Archives of General Psychiatry was funded by Upjohn and had sent the paper to Upjohn to referee. Pierre Simon relates that reports of adverse effects of sulpiride were censored by the French drug company Delagrange. Raymond Battegay, former professor at the Basel Department of Psychiatry, tells of withdrawal of support by Roche of a study comparing moclobemide with cognitive-behavioural psychotherapy: "apparently they feared that psychotherapy would have the same effect as the drug".

And yet, this book is not a diatribe against the pharmaceutical industry. It must be admitted that extensive clinical trials are too expensive to carry out without drug company support. We all depend on the industry to bring money into academia. A study of a drug that reduces platelet aggregation involved 500 researchers and 20000 patients tested for 3 years. It cost Sanofi around \$250 million. No academic or clinical department can compete with Sanofi's budget for research, which, Simon remarks, is larger than that of the whole French INSERM. Although drug companies pay little attention to brain mechanisms, and theory is secondary to the development of drugs, it must also be admitted that much scientific progress has resulted from the use of drugs such as chlorpromazine, imipramine, benzodiazepines and fluoxetine.

Thus, it seems that money, not science, drives pharmacology. What can the medical profession do? A word of advice about training is given by Solomon Snyder of Johns Hopkins University, Maryland: "It is important . . . to come up with new ideas. PhDs are very well trained to be so critical that they criticise their own thoughts, so they're never creative . . . People without such fancy training . . . don't know about that and just try to think new thoughts. My own psychology is if somebody said day is day, I might say maybe day is night, and that attitude always helps". Further advice from other contributors is to stick close to the bedside: it is salutary to note that the novel effects of chlorpromazine and of imipramine were first observed by nurses. The discovery of these and many other drugs was serendipitous of which Simon offers a definition: "A young man looking for a pin in a haystack finds the farmer's daughter... . if the guy is not interested in the farmer's daughter, he has no chance of doing anything". Perhaps the message is that academic psychopharmacologists should search for the farmer's daughter instead of the pin, but get the drug companies to pay for her dowry.

Readers of this book are advised to peruse it piecemeal. To enjoy a stroll in the company of giants, it is best to walk with them one at a time. Those who do so will find many thought-provoking ideas and may begin to question the widespread assumption that there is a drug treatment for every psychiatric illness, whether already recognised or yet to be described. As Professor Battegay disarmingly remarks to his interviewer: "I don't know, Dr. Healy, if psychiatry is really necessary ....".

Heather Ashton Emeritus Professor of Clinical Psychopharmacology, Department of Psychiatry, Royal Victoria Infirmary, Queen Victoria Road, Newcastle uponTyne NEI 4LP, UK

\section{Psychiatric Management in Neurological Disease}

Edited by Edward C. Lauterbach. Washington, DC: American Psychiatric Press. 2000. 346 pp. $€ 31.50$ (hb). ISBN 0880487860

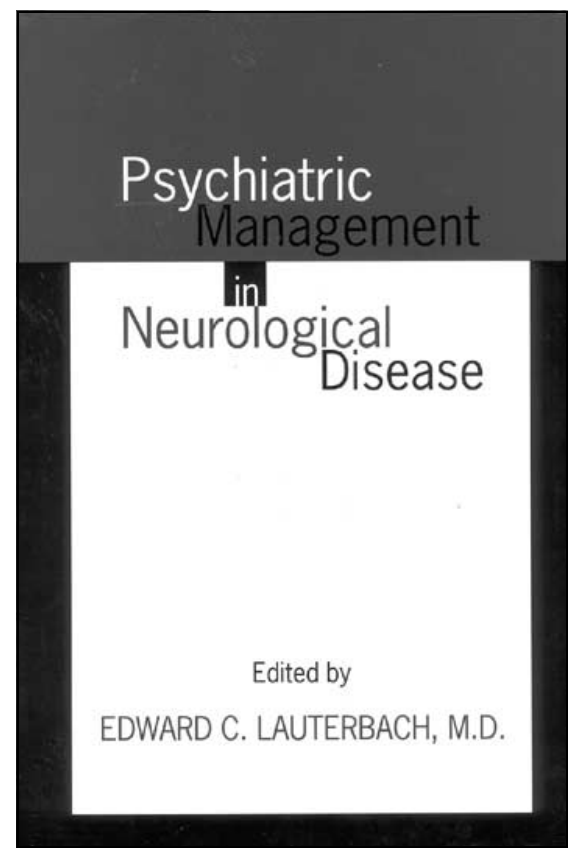

The presence of a psychiatrist on a neurological ward is, of course, no longer novel, but it remains an occurrence that is too infrequent for the full and proper management of many patients with neurological 
disorders. It is to be hoped that this book will encourage psychiatrists to become more aware of their potential for improving the management of patients with a variety of neurological diseases.

This volume is a useful reference of not only the psychiatric management, but also the clinical state and psychiatric complications of selected neurological conditions. The emphasis on the treatment of individual psychiatric problems is welcome and the book forms a worthy introduction to this field. This is a multi-author text, but it is clear that fairly strict editorial control has been exercised, which makes it easy to use in everyday liaison neuropsychiatric practice. There is a helpful initial introduction to the neuropsychiatric presentations of neurological disorder and their possible management, followed by eight chapters on individual syndromes. Each chapter includes an introduction, a brief epidemiological overview, an outline of the neurological presentation, pathology and investigation of the disorder, a review of the psychiatric manifestations, and finally details of management of the neurological and psychiatric problems that might arise from the condition. The only exception to this is the chapter on stroke, in which only the neuropsychiatric aspects are covered. There are several very helpful lists, for instance of drug/drug interactions and of disease/drug interactions. A number of illustrations, including magnetic resonance imaging brain scans and a startling picture of a KayserFleischer ring, augment the text.

The main weaknesses of the book are that the selection of neurological disorders is incomplete and that the coverage of some that are included is variable. There are, for example, 42 pages on Fahr disease but only 22 on multiple sclerosis. Movement disorders are well covered, particularly Parkinson's disease, Huntington's disease, Wilson's disease and dystonia. There is a useful chapter on the management of the psychiatric manifestations and complications of HIV. All of the chapters tend more towards the pharmacological than the psychological or psychotherapeutic management of psychiatric problems in people with these neurological diseases, but the book does end with a short paper about family management issues. Perhaps the emphasis on pharmacological matters indicates a relative paucity of worthwhile research into other areas of psychiatric management, but it is a shame that no attention is paid to the variety of cognitive- behavioural and allied treatments that can be used in this area.

The book does not purport to cover the apparently neurological presentations of psychiatric disorder and makes almost no mention of somatoform disorders. At the outset the editor remarks that books on the psychiatry of Alzheimer's disease and epilepsy exist, and therefore he makes no further mention of them. However, serious omissions appear to be the psychiatric management of migraine, motor neuron disease, infections of the central nervous system other than HIV, normal-pressure hydrocephalus and, possibly, some mention of the sequelae of head injury. Consequently, this cannot by any means be regarded as a comprehensive reference book for those interested in liaison psychiatry on neurological wards, but it can be strongly recommended as a practical handbook for the trainee or general psychiatrist who is consulted by a neurological colleague about a patient with one of the eight conditions featured.

\section{Jonathan Michael Bird Consultant}

Neuropsychiatrist, The Burden Centre for

Neuropsychiatry, Neuropsychology and

Epileptology, Frenchay Hospital, Bristol BSI6 IJB, UK

\section{Guide to Psychiatric Research}

By Arthur Yuwiler \& Lennart Wetterberg. London: CRC (UK) Press. 2000. 152 pp. E33.99 (pb). ISBN 084930295 I

So, you have seen an interesting clinical problem, been prompted to read around the subject and found yourself asking more questions than seem to have answers: a typical starting point for research. What next? Guidance from a venerable and respected senior? A search along the library shelves for the section marked 'research methodology'? Either is entirely reasonable and, in some ways, this slender volume combines the two. Written in a conversational, easy style by authors with a wealth of research and teaching experience, the book promises "to entice people . . . into research". Having developed out of a series of research methodology seminars for medical students, it retains a discursive and thought-provoking approach, spiced up with anecdotes from the history of medical endeavour.

The structure reflects this emphasis, with almost half the book devoted to a chapter on methodology. This contains a good review of subject selection and useful discussion on the different areas of investigation in psychiatric research and on methods used. The authors' background in neurobiological research is evident in their succinct summaries on imaging and neurotransmitters, but there is no overindulgence.

The introductory chapter engages with a wide-ranging discussion on the nature of scientific enquiry and the ethics of research on humans and animals - particularly topical at present. If you are looking for a handy guide to statistics, however, you will not find it here. The authors boldly state that "this is not a textbook on statistics". (Would that they might turn their hand to one - their style and ability to communicate could cut through much that is daunting in existing texts.)

A subsequent 'how to . . .' section covers reading and writing a paper, and writing a grant proposal. Again, it is full of sensible and deceptively simple advice but also contains a curious account of a fictional grants committee reviewing a lovingly written paper. Populated with characters such as Roberta Receptor (the molecular biologist), Zachery Zingo (the ageing but still research-productive chairman) and Sam Shark ("smooth face, slick hair, a toothy smile and a hard, slick mind"), this entertaining endnote suggests a flair for screenplay that might entice Hollywood should the authors ever tire of biobehaviourism. One last point: most sections of the book are followed by links to websites (both North American and

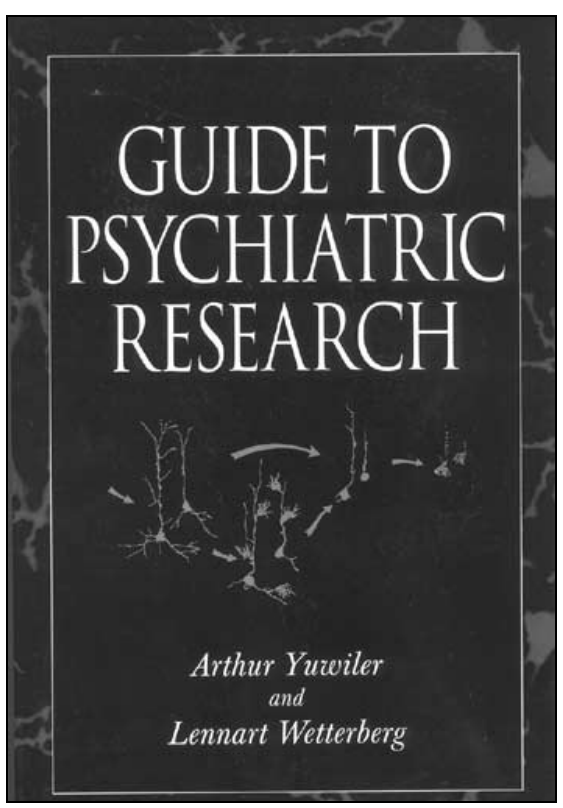

